\title{
Evaluation of Diallel Crosses of Highland Adapted QPM Maize Hybrids under Optimum and Low Nitrogen Conditions
}

Tefera Kumsa $^{1 *}$, Demissew Abakemal ${ }^{1}$, Dufera Tulu ${ }^{1}, Z_{\text {Zeleke Keimiso }}{ }^{1}$, Habtamu Zeleke ${ }^{2}$ and Mekuanent Belay ${ }^{2}$

${ }^{1}$ Ambo Agricultural Research Center, Ethiopia
${ }^{2}$ Haramaya University, Ethiopia

Article History

Received: 26.01.2021

Accepted: 10.02 .2021

Published: 12.03 .2021

Journal homepage:

https://www.easpublisher.com

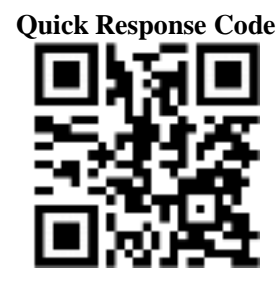

Abstract: Eight inbred lines collected from Ambo Agriculural Research Center Highland maize sub-program and were evaluated under optimum and Low Nitrogen condition at Ambo and Haramaya University in 2018. Breeding programs have created inbred lines of maize introduced from CIMMYT; they were tested locally for their heterosis. The objective of this study was to generate information regarding the combining ability effect of selected highland adapted maize inbred lines and their crosses for further breeding and cultivar development in view of this limitation. P6 was the lines that exhibited positive and hence good combiner for gain yield in all locations and environmental condition and crosses found to be good yield potential in this study were (P1xP2), (P2xP7) and (P4xP6).

Keywords: Diallel, QPM, Breeding program, CIMMYT.

Copyright (C) 2021 The Author(s): This is an open-access article distributed under the terms of the Creative Commons Attribution 4.0 International License (CC BY-NC 4.0) which permits unrestricted use, distribution, and reproduction in any medium for non-commercial use provided the original author and source are credited.

\section{INTRODUCTION}

Maize (Zea mays ssp. mays L) has key importance in assuring the world food security and a high yielding cereal crop as well [1]. In Ethiopia, maize crop has been considered as one of the most important food security assurance and expansion of its agricultural production. According to the Central Statistic Agency report of 2018, maize is an important staple crop, ranking first among cereals in total grain production $(27.43 \%)$ and second in area coverage (16.79\%). However, the national average yield of maize $\left(3.9 \mathrm{t} \mathrm{ha}^{-1)}\right.$ is still low [2] compared to the world average.

In Ethiopia QPM development program was launched in 1994 with the evaluation of open pollinated varieties (OPVs) and pools introduced from CIMMYT [3]. Higher content of lysine and tryptophan have been successfully increased in maize through conventional breeding.

The Ambo highland breeding program is one of the three maize breeding programs under National Maize Research Program of the Ethiopian Institute of Agricultural Research. Yearly, the breeding program handles several numbers of QPM and non QPM crosses generated at different stages of the breeding pipeline with the aim of identifying, superior genotypes for the target agroecolgy. This is because improved commercial maize varieties suited to highland areas of Ethiopia have been fewer and consequently access to maize seed has also been limited. Evaluation of the genetic potential or performances of maize genotypes within the breeding, pipeline will help identifying genotypes with good traits of interest for future use in breeding and cultivar development.

For target environment mating design such as diallel play an important role in the selection o and advancement of breeding materials. Hayman [4] and Griffing [5] proposed the concept of diallel cross as the recombination of genetic variability available in the program, performing crosses among all lineages. The diallel scheme of analysis allows estimating useful genetic information to select parental lines and verify the combining ability effect, which are described as general and specific. The objective of this study was, therefore, to generate information regarding the combining ability effect of selected highland adapted maize inbred lines and their crosses for further breeding and cultivar development in view of this limitation. 


\section{Material ANd Method \\ Study Location}

The study was conducted at Ambo (optimum and Low-N conditions), Agricultural Research Center and Haramaya University in the main cropping season of 2019. The locations represent highland, sub-humid maize growing environments of Ethiopia [6].

\section{Experimental Materials}

Eight QPM inbred lines were selected depending on their performance and diverse pedigree back grounds (Table 1). The experiment was composed of $28 \mathrm{~F} 1$ crosses formed using half diallel mating design at Ambo during the main cropping season of 2019 and Two (2) commercial hybrid checks: check-1 (Kolba) and check-2(Jibat).

Table-1: List of highland QPM inbred lines selected and used for diallel cross formation

\begin{tabular}{|c|c|c|c|}
\hline $\mathbf{S} / \mathbf{N}$ & $\begin{array}{l}\text { Lines } \\
\text { Code }\end{array}$ & Pedigree & Source \\
\hline 1 & L1 & $\begin{array}{l}\text { [ECU/SNSYN[SC/ETO]]c1F1-\#\#(GLS=1)-34-3-1-2/CML144(BC2)-34-8-2-2-1- } \\
\text { 1-\#-1-B-\#-\#-B }\end{array}$ & AHMBP \\
\hline 2 & L2 & [POOL9Ac7-SR(BC2)]FS67-1-2-3-1-\#/CML144(BC2)-10-11-2-4-1-2-\#-\#-B & AHMBP \\
\hline 3 & L3 & [POOL9Ac7-SR(BC2)]FS68-1-1-2-1-1/CML144(BC2)-33-10-2-4-1-2-\#-1-B-\#-B & AHMBP \\
\hline 4 & L4 & $\begin{array}{l}\text { (CML197/(CML197/[(CLQRCWQ50/CML312SR)-2-2-1-BB/CML197]-BB)F2)- } \\
\text { B-B-9-1-B-\# }\end{array}$ & AHMBP \\
\hline 5 & L5 & $\begin{array}{l}\text { (CML197/(CML197/[(CLQRCWQ50/CML312SR)-2-2-1-BB/CML197]-BB)F2)- } \\
\text { B-B-35-2-B-\# }\end{array}$ & AHMBP \\
\hline 6 & L6 & $\begin{array}{l}\text { (CML197/(CML197/[(CLQRCWQ50/CML312SR)-2-2-1-BB/CML197]-BB)F2)- } \\
\text { B-B-44-2-B-\# }\end{array}$ & AHMBP \\
\hline 7 & L7 & (CML197/(CML197/(CLQRCWQ50/CML312SR)-2-2-1-BBB)F2)-B-B-18-2-B-\# & AHMBP \\
\hline 8 & L8 & (CML395/(CML395/CML511)F2)-B-B-37-1-B-\# & AHMBP \\
\hline
\end{tabular}

$*$ AHMBP = Ambo Highland Maize Breeding Program

\section{Experimental Design and Cultural Practices}

In each location alpha lattice experimental design $(5 \times 6)$ were used with two replications. Each plot consisted a single row of $5.25 \mathrm{~m}$ long. The spacing was $75 \mathrm{~cm}$ between rows and $25 \mathrm{~cm}$ between plants. Planting was done in the rainy season of 2019 after reliable moisture level of soil attained to ensure good germination and seedling development using two seeds per hill and thinned out to one plant after 35 days of planting.

\section{Data Collection}

The data were recorded by field scorer and taken mostly from twenty- one maize plants for each plot. Traits such as Grain yield, days to anthesis, days to silking, Plant height, ear height, root and stalk lodging, husk cover, ear rot, disease (TLB and Rust), plant aspect, ear aspect, days to maturity, Ear per plant, number of ear and number of plant were recorded for the study.

Days to $50 \%$ silking and $50 \%$ anthesis were calculated as number of days from sowing until $50 \%$ of plants in each row showing tassel and silks. Anthesissilking interval (ASI; days) computed as the difference between days to $50 \%$ anthesis and silking. Plant height $(\mathrm{PH} ; \mathrm{cm})$ was measured as the distance from the soil surface to the top of tassel. Ear height $(\mathrm{EH} ; \mathrm{cm})$ was measured as the distance from the soil surface to the main ear bearing node. Plant aspect was recorded by observing overall phenotypic appearance of the plant in a plot by using 1 to 5 scoring scale; where $1=$ excellent and $5=$ poor. Ear aspect was recorded by observing overall phenotypic appearance of the ears in a plot at harvesting time by using 1 to 5 scoring scale; where $1=$ excellent and $5=$ poor. Kernel modification data measured by using 1 to 5 scoring scale of opaque's of the kernel. 1=excellent and 5= choky type.

\section{Data Analysis}

ANOVA for individual was conducted using PROC GLM procedure of SAS, version 9.0 [7] to determine the differences among the genotypes.

Further genetic analysis were done for traits that showed statistically significant different among genotypes. F1 diallel crosses were subjected to combining ability analysis using modification of the DIALLEL-SAS program [8].

\section{Results AND Discussion Analysis of Variance (ANOVA)}

Individual location analysis of variances were made on grain yield and yield related traits such as days to anthesis, days to silking, plant and ear height, ear per plant, bad husk cover, ear rot, ear aspect, plant aspect, ear texture, anthesis silking interval, root lodge, (Rust) diseases, stalk lodge and E.turcicum leaf blight (TLB) for each location.

Result in table 2 indicated that mean squares due to entries (genotypes) revealed significant highly $(p<0.01)$ difference for grain yield and other related traits at Ambo (optimum and low-N condition) and Haramaya as well, indicating that there is variability 
between materials evaluated. As the study result showed that significant differences were observed among F1 hybrids for all traits in line with the report of Mohamed Ali, 2020 [16].
Results in Table 2 indicate that mean squares of genotypes and most character in all location and environmental condition were significantly different. Under managed low $\mathrm{N}$ stress $\mathrm{F} 1$ hybrids were significant for most traits. This finding is similar with the report of Susan et al., [9].

Table-2: Mean squares due to genotypes for grain yield and related traits at three locations under optimum and low -N conditions in 2019

\begin{tabular}{|c|c|c|c|c|c|c|}
\hline \multicolumn{9}{|c|}{ Means Squares } \\
\hline \multicolumn{3}{|c|}{ Ambo (Opt.) } & Ambo (Low-N) & \multicolumn{3}{c|}{ Haramaya (Opt) } \\
\hline Genotypes & Error & Genotypes & Error & Genotype & Error & \\
\hline Traits & DF=29 & DF=18 & DF=29 & DF=18 & DF=29 & DF=18 \\
\hline GY & $4.30^{* *}$ & $0.80^{* *}$ & $0.82^{* *}$ & $0.43^{*}$ & $9.20^{* *}$ & $1.43^{* *}$ \\
\hline AD & $26.41^{* *}$ & $8.21^{* *}$ & $21.60^{* *}$ & $3.41^{*}$ & $84.5^{\mathrm{NS}}$ & $97.6^{\mathrm{NS}}$ \\
\hline ASI & $18.23^{*}$ & $6.04^{*}$ & $26.1^{*}$ & $10.1^{* *}$ & $0.38^{\mathrm{NS}}$ & $0.29^{\mathrm{NS}}$ \\
\hline EPP & $0.8^{* *}$ & $0.02^{* *}$ & $0.11^{\mathrm{NS}}$ & $0.08^{\mathrm{NS}}$ & $0.08^{* *}$ & $0.02^{* *}$ \\
\hline HC & $396.4^{* *}$ & $122.4^{* *}$ & $5.91^{\mathrm{NS}}$ & $7.02^{\mathrm{NS}}$ & $1830.6^{*}$ & 670.4 \\
\hline EA & $0.3^{* *}$ & $0.04^{* *}$ & $0.28^{* *}$ & $0.1^{* *}$ & $0.34^{* *}$ & $0.1^{* *}$ \\
\hline PA & $0.4^{* *}$ & $0.51^{* *}$ & $0.41^{* *}$ & $0.2^{*}$ & $0.3^{* *}$ & $0.1^{* *}$ \\
\hline TXT & $0.29^{\mathrm{NS}}$ & $0.21^{*}$ & $0.42^{* *}$ & $012^{* *}$ & ---- & ---- \\
\hline CV & 18.7 & & 41.9 & & 15.9 & \\
\hline GY mean & 4.8 & & 1.5 & & 7.5 & \\
\hline
\end{tabular}

$* *=$ highly significant at $(\mathrm{p}<0.01), *=$ significant at $(\mathrm{p}<0.05), \mathrm{GY}=$ grain yield, $\mathrm{AD}=$ number of days to anthesis, ASI=anthesis silking interval, $\mathrm{EPP}=$ number of ears per plant, $\mathrm{HC}=$ husk cover, $\mathrm{EA}=$ ear aspect, $\mathrm{PA}=$ plant aspect, $\mathrm{TXT}=$ seed texture

(Modification) Opt.=optimum condition, Low $-\mathrm{N}=$ low nitrogen condition

\section{Combining ability analysis}

Analysis of variances for combining ability revealed that variances for general combining ability (GCA) and specific combining ability (SCA) were significant for some traits while non-significant for most traits under the three environmental conditions and its similar with the finding of [10].
Traits like ear rot were revealed significant variation for GCA at Haramaya site. At Ambo (optimum condition) traits that showed significant variation for GCA were days to tasseling and plant aspect, whereas at Ambo (Low N condition) husk cover and ear aspect were revealed significant for GCA.

Table-3: Diallel analysis of variance for yield and yield related traits of highland maize hybrids grown at Ambo (optimum and Low -N) and Haramaya

\begin{tabular}{|c|c|c|c|c|c|c|c|c|c|}
\hline \multirow{2}{*}{ Source I } & \multicolumn{9}{|c|}{ Mean squares } \\
\hline & & $\overline{\mathrm{DA}}$ & DS & ASI & $\mathrm{HC}$ & PA & $\mathrm{Kmo}$ & EA & (Yield (t ha-1) \\
\hline \multicolumn{10}{|c|}{ Ambo (opt) } \\
\hline Crosses & 27 & 21.4 & 1.4 & 11.8 & 110.3 & 0.28 & $0.3^{*}$ & $0.31^{*}$ & 3.3 \\
\hline GCA & 7 & $35.3^{*}$ & $2.1^{*}$ & 12.6 & 150.8 & $0.3^{*}$ & 0.2 & 0.1 & 0.8 \\
\hline $\mathrm{SCA}$ & 20 & 22.4 & 1.5 & 12.1 & $96.9 *$ & 0.4 & $0.3^{*}$ & 0.2 & 4.5 \\
\hline Error & 22 & 13.1 & 25.2 & 16.11 & 106.3 & 0.2 & 0.2 & 0.2 & 2.9 \\
\hline \multicolumn{7}{|c|}{ Ambo(Low N) } & \multicolumn{3}{|l|}{ SEN } \\
\hline Crosses & 27 & 1.1 & 15 & 0.6 & 0.2 & $0.3^{*}$ & $0.3^{*}$ & 0.22 & 0.6 \\
\hline GCA & 20 & 1.2 & 18.4 & 0.8 & $0.3^{*}$ & 0.2 & 2.0 & $0.3^{*}$ & 0.9 \\
\hline $\mathrm{SCA}$ & 7 & 0.9 & 13.8 & 0.5 & 0.2 & $0.3^{*}$ & 3.3 & 0.23 & 0.6 \\
\hline Error & 22 & 15.0 & 15 & 27.7 & 0.3 & 0.29 & 2.7 & 0.19 & 0.5 \\
\hline \multicolumn{7}{|c|}{ Haramaya } & \multicolumn{3}{|c|}{$\mathrm{ER}$} \\
\hline Crosses & 27 & 68.8 & 69.5 & $0.31^{*}$ & $187.5^{*}$ & 0.19 & $172.1^{*}$ & $* 0.29 *$ & 2.5 \\
\hline GCA & 7 & 130.2 & 127.1 & 0.16 & 162.3 & 0.18 & $216.3^{*}$ & $* 0.19$ & 0.8 \\
\hline $\mathrm{SCA}$ & 20 & 80.1 & 83.2 & 0.4 & $195.7 * *$ & $* 0.2$ & 156.6 & $0.28 *$ & 3.1 \\
\hline Error & 22 & 190.1 & 184.9 & 0.4 & 699.4 & 0.19 & 83.5 & 0.14 & 3.2 \\
\hline
\end{tabular}

*Significant at $\mathrm{P}<0.05$. ** Significant at $\mathrm{P}<0.01$. GCA, general combining ability; SCA, specific combining ability; DT, days to tasseling; DS, days to silking; ASI, anthesis silking interval; PA, plant aspect; Kmod,kernel modification; EA, ear aspect; HC, husk cover and GY, grain yield, SEN, senesces , ER , ear rot 


\section{General Combining Ability (GCA) Effects}

Estimates of general combining ability effects are presented in Table (4). Some inbred parents showed positive and significant GCA effects for some traits at Ambo (optimum and Low- N conditions) site. Parents with significantly desirable GCA effects were considered as high combiners, Low or poor combiners had significant but negative (undesirable) GCA effect fpr grain yield [11]. At Ambo (opt.) the good general combiners for major yield determining characters were
P2, P4, P5, P6 and P7, while at Low-N condition P1 and P8 were good combiner or had good per se performance. At Haramaya inbred lines such as P3 and P6 showed positive gca effect for gain yield thought not significant. Lines with Positive gca estimate have genes that contribute to the increment of yield and the GCA effects represent the additive nature of gene action and a good general combiner parent is characterized by its better breeding value when crossed with other parentsas stated in the in the report of Kumar et al., [12].

Table-4: Estimates for general combining ability effects of inbred parents

Ambo (opt)

\begin{tabular}{|r|c|c|c|c|c|c|c|c|}
\hline Parents & DA & DS & ASI & PA & Kmod & HC & EA & GY \\
\hline SE(gi) & 0.69 & 0.9 & 0.7 & 0.08 & 0.07 & 1.9 & 0.09 & 0.32 \\
\hline \multicolumn{8}{|c|}{ Ambo(Low N) } \\
\hline Parents & DA & DS & ASI & PA & SEN & HC & EA & GY \\
\hline P1 & -0.75 & -2.23 & 1.48 & -0.10 & 0.00 & -0.16 & 0.06 & $0.32^{*}$ \\
\hline P2 & -1.00 & $-2.81^{*}$ & 1.81 & 0.10 & -0.33 & -0.16 & 0.10 & 0.01 \\
\hline P3 & 0.50 & 1.69 & -1.19 & $-0.23^{*}$ & -0.42 & 0.79 & $0.23^{*}$ & $-0.38^{*}$ \\
\hline P4 & -0.92 & 0.10 & -1.02 & 0.02 & -0.17 & -0.60 & -0.06 & 0.14 \\
\hline P5 & -1.25 & $-2.73^{*}$ & 1.48 & 0.01 & 0.50 & -0.60 & 0.10 & $-0.36^{*}$ \\
\hline P6 & $1.58^{*}$ & $2.77^{*}$ & -1.19 & 0.06 & 0.58 & -0.04 & -0.15 & 0.02 \\
\hline P7 & $2.00^{*}$ & 2.02 & -0.02 & -0.06 & 0.00 & 0.79 & -0.16 & -0.08 \\
\hline P8 & -0.17 & 1.19 & -1.35 & 0.06 & -0.17 & -0.04 & $-0.23^{*}$ & $0.35^{*}$ \\
\hline SE(gi) & 0.74 & 1.17 & 1.00 & 0.10 & 0.31 & 0.47 & 0.08 & 0.14 \\
\hline
\end{tabular}

Ambo (Low N)

\begin{tabular}{|r|c|c|c|c|c|c|c|c|}
\hline Parents & DA & DS & ASI & PA & SEN & HC & EA & GY \\
\hline P1 & -0.75 & -2.23 & 1.48 & -0.10 & 0.00 & -0.16 & 0.06 & $0.32^{*}$ \\
\hline P2 & -1.00 & $-2.81^{*}$ & 1.81 & 0.10 & -0.33 & -0.16 & 0.10 & 0.01 \\
\hline P3 & 0.50 & 1.69 & -1.19 & $-0.23^{*}$ & -0.42 & 0.79 & $0.23^{*}$ & $-0.38^{*}$ \\
\hline P4 & -0.92 & 0.10 & -1.02 & 0.02 & -0.17 & -0.60 & -0.06 & 0.14 \\
\hline P5 & -1.25 & $-2.73^{*}$ & 1.48 & 0.01 & 0.50 & -0.60 & 0.10 & $-0.36^{*}$ \\
\hline P6 & $1.58^{*}$ & $2.77^{*}$ & -1.19 & 0.06 & 0.58 & -0.04 & -0.15 & 0.02 \\
\hline P7 & $2.00^{*}$ & 2.02 & -0.02 & -0.06 & 0.00 & 0.79 & -0.16 & -0.08 \\
\hline P8 & -0.17 & 1.19 & -1.35 & 0.06 & -0.17 & -0.04 & $-0.23^{*}$ & $0.35^{*}$ \\
\hline SE(gi) & 0.74 & 1.17 & 1.00 & 0.10 & 0.31 & 0.47 & 0.08 & 0.14 \\
\hline
\end{tabular}

\begin{tabular}{|c|c|c|c|c|c|c|c|c|}
\hline \multicolumn{9}{|c|}{ Haramaya (Opt) } \\
\hline Parent & DA & DS & ASI & PA & ER & $\mathrm{HC}$ & EA & Gy \\
\hline P1 & 0.35 & 0.38 & -0.02 & -0.17 & $-6.55^{*}$ & $-14.29 *$ & -0.10 & -0.16 \\
\hline $\mathrm{P} 2$ & -2.31 & -2.21 & -0.10 & 0.08 & $-4.56^{*}$ & $-11.49 *$ & $-0.19 *$ & -0.15 \\
\hline P3 & 2.02 & 1.96 & 0.06 & 0.13 & -1.17 & 1.25 & 0.06 & 0.58 \\
\hline $\mathrm{P} 4$ & 1.44 & 1.46 & -0.02 & 0.08 & 2.46 & $12.24 *$ & 0.10 & -0.31 \\
\hline P5 & 2.60 & 2.71 & -0.10 & 0.00 & 0.19 & $11.99 *$ & 0.06 & -0.09 \\
\hline P6 & $-5.65 *$ & $-5.71 *$ & 0.06 & 0.08 & $7.20 *$ & $14.27 *$ & $0.19 *$ & 0.08 \\
\hline P7 & -2.73 & -2.63 & -0.10 & 0.00 & 1.58 & -4.10 & -0.10 & 0.01 \\
\hline P8 & 4.27 & 4.04 & 0.23 & $-0.21 *$ & 0.84 & -9.87 & -0.02 & 0.04 \\
\hline SE(gi) & 2.63 & 2.59 & 0.12 & 0.08 & 1.74 & 5.04 & 0.07 & 0.34 \\
\hline
\end{tabular}

\section{Specific Combining Ability (SCA) Effects}

Results of the SCA effects of the crosses for yield and different yield related characters are presented in Table 5. Positive SCA effect for grain yield was observed in 14 crosses but significant positive effects were observed on three crosses for gain yield as well.
For traits such as days to silking and plant aspect, the cross (P1xP7) showed significant negative SCA effect, but positive and significant effect for the trait kernel modification from the same crosses at this location. 
At Ambo Low-N condition, 12 F1 crosses revealed positive SCA effect for grain yield, of these crosses (P1xP3) and (P3xP8) showed significant positive SCA effect for gain yield. From (P2xP5) positive and significant SCA effect observed for the traits Senesces but significant negative effect for days to anthesis. At Haramaya 12 crosses revealed positive SCA effect for gain yield thought not significant.The cross of (P3 x P) and (P5 x P6) exhibited significant SCA effect for traits husk cover, ear rot and ear aspect indicating tolerant to open tip and rotting. This finding is in line with the report of in line with the report of Francis et al., [13]. At all testing and environments crosses that exhibited positive SCA effect for gain yield indicating the prevalence of non-additive gene effects for the inheritance of these traits as the report of Motiar et al., [14]. Significant SCA effects indicated that the crosses performed better or poorer than what would be expected based on GCA effects of the respective parent as the report of Bitew et al., [15].

Table-5: Estimates of specific combining ability (SCA) effects on yield and its components in diallel cross of maize

\begin{tabular}{|c|c|c|c|c|c|c|c|c|}
\hline \multicolumn{9}{|c|}{ Ambo (opt) } \\
\hline Crosses & $D A$ & $D S$ & $A S I$ & $P A$ & Kmod & $H C$ & $E A$ & $G Y$ \\
\hline P1xP2 & 1.40 & 0.26 & 1.14 & $0.71 * *$ & $-0.54 *$ & -4.84 & 0.35 & $-2.06^{*}$ \\
\hline P1xP3 & 1.32 & 1.76 & -0.44 & 0.01 & -0.12 & -1.00 & -0.40 & 0.66 \\
\hline P1xP4 & 2.82 & 5.10 & -2.27 & 0.34 & 0.05 & 1.71 & 0.05 & -0.29 \\
\hline P1xP5 & $5.15^{*}$ & $7.51 *$ & -2.36 & 0.38 & -0.29 & -0.59 & 0.43 & 0.22 \\
\hline P1xP6 & -3.26 & -2.90 & -0.36 & -0.37 & 0.21 & 5.59 & -0.20 & 1.00 \\
\hline P1xP7 & -2.85 & $-6.74 *$ & 3.89 & $-0.54 *$ & $0.84 * *$ & -4.63 & -0.07 & -0.35 \\
\hline P1xP8 & -4.60 & -4.99 & 0.39 & $-0.54 *$ & -0.16 & 3.77 & -0.15 & 0.83 \\
\hline P2xP3 & -0.35 & -1.57 & 1.23 & -0.24 & $0.55 *$ & -4.79 & 0.01 & -0.19 \\
\hline $\mathrm{P} 2 \times \mathrm{P} 4$ & -2.35 & $-6.74 *$ & $4.39 *$ & -0.41 & 0.46 & -2.84 & -0.03 & 0.52 \\
\hline $\mathrm{P} 2 \mathrm{xP5}$ & -1.01 & 0.18 & -1.19 & 0.13 & -0.37 & -7.04 & 0.10 & -1.53 \\
\hline P2xP6 & -1.93 & 1.26 & -3.19 & -0.37 & -0.37 & 8.15 & -0.03 & $2.15^{*}$ \\
\hline $\mathrm{P} 2 \mathrm{xP7}$ & -0.01 & 2.93 & -2.94 & -0.04 & 0.01 & $11.58^{*}$ & -0.15 & 0.11 \\
\hline $\mathrm{P} 2 \mathrm{xP} 8$ & $4.24 *$ & 3.68 & 0.56 & 0.21 & 0.26 & -0.22 & -0.24 & 1.01 \\
\hline P3xP4 & -3.43 & -5.74 & 2.31 & -0.37 & 0.13 & -0.74 & -0.03 & -0.27 \\
\hline P3xP5 & 0.90 & 2.68 & -1.77 & 0.42 & -0.20 & 6.81 & 0.35 & -1.27 \\
\hline P3xP6 & 3.49 & 2.26 & 1.23 & 0.42 & $-0.45^{*}$ & -6.86 & 0.22 & -1.74 \\
\hline $\mathrm{P} 3 \times \mathrm{P} 7$ & $-4.10^{*}$ & -0.57 & -3.52 & -0.24 & 0.17 & 4.52 & -0.15 & $2.68 *$ \\
\hline P3xP8 & 2.15 & 1.18 & 0.98 & 0.01 & -0.08 & 2.07 & 0.01 & 0.14 \\
\hline $\mathrm{P} 4 \mathrm{xP5}$ & 0.40 & 0.01 & 0.39 & 0.01 & -0.29 & -4.04 & -0.20 & -0.22 \\
\hline P4xP6 & -2.51 & -0.90 & -1.61 & -0.24 & $0.21 * *$ & $11.25^{*}$ & -0.07 & 1.31 \\
\hline P4xP7 & $5.40 *$ & $6.76^{*}$ & -1.36 & $0.59 *$ & -0.41 & $-6.87 *$ & 0.55 & -1.80 \\
\hline P4xP8 & -0.35 & 1.51 & -1.86 & 0.09 & -0.16 & 1.53 & $-0.28^{*}$ & 0.76 \\
\hline P5xP6 & 1.32 & -0.99 & 2.31 & -0.20 & $0.38 * *$ & -5.55 & -0.20 & -0.28 \\
\hline P5xP7 & -2.76 & $-6.32 *$ & 3.56 & -0.37 & 0.01 & $10.68 *$ & -0.32 & $2.09 *$ \\
\hline P5xP8 & $-4.01 *$ & -3.07 & -0.94 & -0.37 & 0.76 & -0.27 & -0.15 & 1.00 \\
\hline P6xP7 & 2.32 & 1.76 & 0.56 & 0.38 & 0.01 & $-10.49 *$ & -0.20 & -0.71 \\
\hline P6xP8 & 0.57 & -0.49 & 1.06 & 0.38 & 0.01 & -2.09 & 0.47 & -1.72 \\
\hline $\mathrm{P} 7 \mathrm{xP8}$ & 1.99 & 2.18 & -0.19 & 0.21 & -0.62 & -4.80 & 0.35 & $-2.01 *$ \\
\hline$S E(i j)$ & 2.16 & 2.99 & 2.39 & 0.27 & 0.23 & 6.16 & 0.29 & 1.02 \\
\hline
\end{tabular}

Ambo (Low N)

\begin{tabular}{|c|c|c|c|c|c|c|c|c|}
\hline Crosses & $\boldsymbol{D A}$ & $\boldsymbol{D S}$ & $\boldsymbol{A S I}$ & $\boldsymbol{P A}$ & $\boldsymbol{S E N}$ & $\boldsymbol{H C}$ & $\boldsymbol{E A}$ & $\boldsymbol{G} \boldsymbol{~}$ \\
\hline P1xP2 & -2.75 & -0.51 & -2.24 & -0.41 & -0.88 & 2.45 & $-0.54^{*}$ & $0.99^{*}$ \\
\hline P1xP3 & $5.25^{*}$ & 2.99 & 2.26 & $0.84^{*}$ & $2.20^{*}$ & -1.15 & 0.33 & $-1.18^{* *}$ \\
\hline P1xP4 & 0.67 & 3.57 & -2.90 & -0.33 & -1.05 & 0.24 & -0.38 & 0.44 \\
\hline P1xP5 & 1.00 & -4.10 & 5.10 & 0.17 & 0.29 & 0.24 & 0.46 & -0.41 \\
\hline P1xP6 & -0.33 & -0.60 & 0.26 & 0.34 & 0.70 & -0.32 & 0.46 & -0.22 \\
\hline P1xP7 & 0.75 & 2.15 & -1.40 & -0.37 & -0.21 & -1.15 & -0.13 & -0.19 \\
\hline P1xP8 & -4.58 & -3.51 & -1.07 & -0.24 & -1.05 & -0.32 & -0.21 & 0.57 \\
\hline P2xP3 & 0.50 & -0.93 & 1.43 & 0.13 & 0.54 & -1.15 & 0.04 & -0.37 \\
\hline P2xP4 & 0.92 & 1.15 & -0.24 & 0.21 & 0.29 & 0.24 & 0.08 & -0.46 \\
\hline P2xP5 & -2.25 & $-7.51^{*}$ & 5.26 & 0.21 & $2.12^{*}$ & 0.24 & 0.17 & -0.16 \\
\hline P2xP6 & 1.42 & 1.99 & -0.57 & 0.13 & -0.46 & -0.32 & -0.08 & -0.21 \\
\hline P2xP7 & 2.00 & 2.74 & -0.74 & 0.17 & -0.88 & -1.15 & $0.58^{*}$ & 0.35 \\
\hline P2xP8 & 0.17 & 3.07 & -2.90 & -0.45 & -0.71 & -0.32 & -0.25 & -0.15 \\
\hline P3xP4 & -0.58 & -2.35 & 1.76 & -0.04 & -0.63 & -0.71 & -0.04 & 0.03 \\
\hline P3xP5 & 2.75 & 5.49 & -2.74 & -0.04 & 0.70 & -0.71 & -0.21 & 0.25 \\
\hline P3xP6 & $-3.08^{*}$ & -0.01 & -3.07 & $-0.62^{*}$ & -1.38 & -1.27 & 0.04 & 0.57 \\
\hline
\end{tabular}


Tefera Kumsa et al., EAS J Biotechnol Genet; Vol-3, Iss-2 (Mar-Apr, 2021): 30-37

\begin{tabular}{|c|c|c|c|c|c|c|c|c|}
\hline P3xP7 & -3.50 & -3.76 & 0.26 & -0.08 & -0.80 & $6.25^{*}$ & -0.04 & $0.77^{*}$ \\
\hline P3xP8 & -1.33 & -1.43 & 0.10 & -0.20 & -0.63 & -1.27 & -0.13 & -0.07 \\
\hline P4xP5 & -0.33 & 2.07 & -2.40 & 0.05 & -0.55 & 0.68 & 0.08 & 0.35 \\
\hline P4xP6 & -1.67 & -1.43 & -0.24 & -0.29 & -0.63 & 0.13 & -0.17 & 0.46 \\
\hline P4xP7 & -2.08 & -5.18 & 3.10 & 0.26 & 1.45 & -0.71 & -0.25 & -0.41 \\
\hline P4xP8 & 3.08 & 2.15 & 0.93 & 0.13 & 1.12 & 0.13 & $0.67^{*}$ & -0.41 \\
\hline P5xP6 & 1.67 & 1.90 & -0.24 & -0.29 & -1.30 & 0.13 & -0.08 & -0.13 \\
\hline P5xP7 & -1.75 & 2.15 & -3.90 & -0.49 & $-1.71^{*}$ & -0.71 & -0.42 & 0.11 \\
\hline P5xP8 & -1.08 & -0.01 & -1.07 & 0.38 & 0.45 & 0.13 & 0.00 & -0.01 \\
\hline P6xP7 & 1.42 & 0.15 & 1.26 & 0.42 & $2.20^{*}$ & -1.27 & 0.08 & -0.57 \\
\hline P6xP8 & 0.58 & -2.01 & 2.60 & 0.30 & 0.87 & $2.92^{*}$ & -0.25 & 0.11 \\
\hline P7xP8 & 3.17 & 1.74 & 1.43 & 0.09 & -0.05 & -1.27 & 0.17 & -0.05 \\
\hline SE $(\boldsymbol{i j})$ & 2.30 & 3.66 & 3.14 & 0.32 & 0.97 & 1.49 & 0.26 & 0.43 \\
\hline
\end{tabular}

Haramaya(opt)

\begin{tabular}{|c|c|c|c|c|c|c|c|c|}
\hline Crosses & $D A$ & $D S$ & $A S I$ & $P A$ & $H C$ & $E R$ & $\boldsymbol{E A}$ & $G Y$ \\
\hline P1xP2 & -0.02 & 0.23 & -0.25 & -0.27 & 6.79 & 26.3 & 0.20 & 1.13 \\
\hline P1xP3 & -3.36 & -3.44 & 0.08 & 0.43 & 1.21 & -3.11 & 0.20 & -1.08 \\
\hline $\mathrm{P} 1 \times \mathrm{xP} 4$ & -3.27 & -3.44 & 0.17 & -0.02 & 6.13 & 10.3 & $0.41 *$ & -0.08 \\
\hline P1xP5 & -3.94 & -4.19 & 0.25 & -0.44 & -2.31 & -17.7 & -0.30 & 1.11 \\
\hline P1xP6 & 3.81 & 4.23 & -0.42 & 0.23 & -9.32 & -26.1 & -0.17 & -1.40 \\
\hline P1xP7 & 1.89 & 1.64 & 0.25 & -0.19 & 0.46 & 5.3 & -0.38 & 1.15 \\
\hline P1xP8 & 4.89 & 4.98 & -0.08 & 0.27 & -2.96 & 5.11 & 0.04 & -0.82 \\
\hline P2xP3 & 8.81 & 9.14 & -0.33 & -0.07 & -0.28 & -12.9 & $-0.46^{*}$ & 0.86 \\
\hline $\mathrm{P} 2 \mathrm{xP} 4$ & 8.89 & 9.14 & -0.25 & -0.27 & -6.57 & -20.3 & -0.51 & 0.33 \\
\hline $\mathrm{P} 2 \mathrm{xP5}$ & 3.23 & 3.39 & -0.17 & 0.31 & 1.35 & 10.01 & 0.04 & -1.10 \\
\hline P2xP6 & $-15.02 *$ & $-15.7 *$ & 0.67 & 0.23 & $-1.91 *$ & -28.9 & $0.41 *$ & 1.10 \\
\hline P2xP7 & -6.44 & -6.77 & 0.33 & -0.19 & $0.32 *$ & 30.64 & -0.05 & 1.14 \\
\hline P2xP8 & 0.56 & 0.56 & $0.00 *$ & 0.27 & 0.30 & -4.8 & 0.37 & -1.26 \\
\hline P3xP4 & -2.94 & -2.52 & -0.42 & 0.43 & -0.25 & 50.6 & 0.24 & -1.20 \\
\hline P3xP5 & -4.11 & -3.77 & -0.33 & 0.02 & -2.93 & 4.71 & 0.04 & -0.65 \\
\hline P3xP6 & 3.14 & 3.14 & 0.00 & $-0.57 *$ & $-9.69 *$ & $-29.9 *$ & $-0.59 *$ & 0.64 \\
\hline P3xP7 & 1.73 & 1.56 & 0.17 & 0.27 & 15.53 & $8.2 * *$ & 0.45 & -0.05 \\
\hline P3xP8 & -3.27 & -4.11 & $0.83 *$ & $-0.52 *$ & -3.58 & -17.53 & 0.12 & 1.48 \\
\hline $\mathrm{P} 4 \times \mathrm{P} 5$ & -7.52 & -7.77 & 0.25 & 0.06 & $-6.07 *$ & -47.23 & -0.26 & -0.73 \\
\hline $\mathrm{P} 4 \mathrm{xP} 6$ & 4.23 & 3.64 & 0.58 & -0.02 & $15.9 *$ & $44.2 *$ & 0.37 & 0.43 \\
\hline P4xP7 & 4.81 & 5.06 & -0.25 & 0.06 & $-5.35 *$ & -30.9 & -0.09 & -0.42 \\
\hline $\mathrm{P} 4 \mathrm{xP} 8$ & -4.19 & -4.11 & -0.08 & -0.23 & -3.82 & -6.72 & -0.17 & 1.67 \\
\hline P5xP6 & 7.56 & 7.39 & 0.17 & 0.06 & 14.49 & $66.4 * *$ & $0.41 *$ & 1.36 \\
\hline P5xP7 & 4.64 & 4.31 & 0.33 & 0.14 & -0.08 & -9.04 & $0.45^{*}$ & -1.23 \\
\hline P5xP8 & 0.14 & 0.64 & -0.50 & -0.15 & -4.45 & -7.11 & -0.38 & 1.25 \\
\hline P6xP7 & -6.11 & -5.27 & $-0.83^{*}$ & -0.19 & $-17.4^{*}$ & $-30.4 * *$ & $-0.42^{*}$ & 0.90 \\
\hline P6xP8 & 2.39 & 2.56 & -0.17 & 0.27 & 7.94 & 4.76 & -0.01 & -0.83 \\
\hline P7xP8 & -0.52 & -0.52 & 0.00 & 0.10 & 6.57 & 26.27 & 0.04 & -1.49 \\
\hline SE(ij) & 8.24 & 8.12 & 0.38 & 0.26 & 5.46 & 15.80 & 0.22 & 1.07 \\
\hline
\end{tabular}

Table-6: Mean square due to combined analyses of genetic variance for grain yield and agronomic traits of maize

\begin{tabular}{|l|l|l|l|l|l|l|l|}
\hline \multicolumn{1}{|c|}{ Source } & df & \multicolumn{1}{c|}{ Yield } & \multicolumn{1}{c|}{ KPE } & \multicolumn{1}{c|}{ EL } & \multicolumn{1}{c|}{ ED } & HC & \multicolumn{1}{c|}{ ER } \\
\hline ENV & 2 & $134.02^{* *}$ & 0.09 & 4.66 & $2.54^{* *}$ & 14.06 & $57.26^{* *}$ \\
\hline REP (ENV) & 6 & 5.64 & $13.88^{*}$ & 3.68 & $0.57^{* *}$ & 17.96 & $64.02^{*}$ \\
\hline Crosses & 65 & 5.52 & $32.64^{* *}$ & $16.22^{* *}$ & $0.2^{* *}$ & $204.91^{* *}$ & $197.12^{* *}$ \\
\hline Crosses*Env & 130 & 5.63 & $9.95^{*}$ & $6.22^{*}$ & $0.12^{*}$ & $73.55^{* *}$ & $69.53^{* *}$ \\
\hline GCA & 11 & $9.81^{*}$ & $22.38^{* *}$ & $10.31^{* *}$ & $0.27^{* *}$ & $157.96^{* *}$ & $114.73^{* *}$ \\
\hline SCA & 54 & 4.65 & $34.74^{* *}$ & $17.42^{* *}$ & $0.18^{* *}$ & $214.48^{* *}$ & $213.90^{* *}$ \\
\hline GCA * ENV & 22 & $8.31^{*}$ & 1.72 & 2.67 & $0.17^{* *}$ & 9.07 & $20.30^{*}$ \\
\hline SCA * ENV & 108 & 5.42 & 1.36 & 2.07 & 0.09 & 4.41 & 7.61 \\
\hline Error & 528 & 5.26 & 6.29 & 4.2 & 0.1 & 33.32 & 33.74 \\
\hline Mean & & 7.34 & 31.25 & 14.66 & 4.49 & 9.48 & 9.11 \\
\hline CV & & 18.63 & 8.03 & & 7.20 & 45.88 & 43.69 \\
\hline
\end{tabular}




\section{CONCLUSION}

In conclusion, inbred lines such as $\mathrm{P} 2, \mathrm{P} 4, \mathrm{P} 5$, P6 and P7 had positive but non-significant GCA effect for grain yield at Ambo optimum condition. P2, P4 and P6 were significant positive GCA effect while, P1 and P8 showed significant positive GCA for gain yield at Ambo low N condition. At Haramaya P3, P6. P7 and P8 had positive but insignificant GCA effect for gain yield. Therefore, P6 was the lines that exhibited positive and hence good combiner for gain yield in all locations and environmental condition. For SCA effects the crosses of those line were selected for further breeding purpose. Positive SCA effects observed from cross such as (P2xP4), (P2xP6), (P2xP7), (P3xP8), (P4xP) 8 and (P5xP) both at Ambo (opt.) and Haramya. At Ambo (optimum and Low $-\mathrm{N}$ ) condition cross that revealed positive SCA effects were (P1xP8), (P2xP7), (P3xP7), $(\mathrm{P} 4 \mathrm{xP} 6)$ and $(\mathrm{P} 5 \mathrm{xP} 7)$. In the same pattern the cross that exhibited positive SCA effect in both Ambo low-N and Haramaya were $(\mathrm{P} 1 \times \mathrm{P} 2),(\mathrm{P} 2 \times \mathrm{P} 7)$ and $(\mathrm{P} 4 \times \mathrm{P} 6)$. The overall study indicated cross that showed positive SCA effects in all testing and environment are (P2xP7) and ( $\mathrm{P} 4 \mathrm{x}$ P6). Therefore those crosses with per se performance could be more rewarding in a hybrid breeding program in the future career.

\section{REFERENCES}

1. Rohman, M. M., Islam, M. R., Naznin, T., Omy, S. H., Begum, S., Alam, S. S., ... \& Hasanuzzaman, M. (2019). Maize production under salinity and drought conditions: Oxidative stress regulation by antioxidant defense and glyoxalase systems. In Plant Abiotic Stress Tolerance (pp. 1-34). Springer, Cham.

2. CSA (Central Statistics Agency). (2018). Agricultural Sample Survey 2017/2018: Report on Area and Production of Major Crops ((Private Peasant Holdings, Meher Season).

3. Teklewold, A., Gissa, D. W., Tadesse, A., Tadesse, B., Bantte, K., Friesen, D., \& Prasanna, B. M. (2015). Quality Protein Maize (QPM): A guide to the technology and its promotion in Ethiopia.

4. Hayman, B. I. (1954). The theory and analysis of diallel crosses. Genetics, 39, 789-809.

5. Griffing, B. R. U. C. E. (1956). Concept of general and specific combining ability in relation to diallel crossing systems. Australian journal of biological sciences, 9(4), 463-493.

6. Worku, M., Bänziger, M., Friesen, D., Horst, W. J., \& Vivek, B. (2008). Relative importance of general combining ability and specific combining ability among tropical maize (Zea mays L.) inbreds under contrasting nitrogen environments.

7. Bullo, N., \& Dagne, W. (2016). Combining ability of inbred lines in quality protein maize (QPM) for varietal improvement. International journal of plant breeding and crop Science, 3(1), 079-089.

8. Bullo, N., \& Dagne, W. (2016). Combining ability of inbred lines in quality protein maize (QPM) for varietal improvement. International journal of plant breeding and crop Science, 3(1), 079-089.

9. Njeri, S. G., Makumbi, D., Warburton, M. L., Jumbo, M. B., \& Chemining'wa, G. (2017). Genetic analysis of tropical quality protein maize (Zea mays L.) germplasm. Euphytica, 213(11), 119.

10. Fayed, E. A., Lelah, A. A. A., Mostafa, E. S. E., \& Morgan, S. F. (2019). Morphological identification and evaluate the combining ability and heterosis of some inbred lines of maize and its crosses. Zagazig Journal of Agricultural Research,46(6), 21452157.

11. Amiruzzaman, M., Islam, M. A., Hassan, L., \& Rohman, M. M. (2010). Combining ability and heterosis for yield and component characters in maize. Academic Journal of Plant Sciences, 3(2), 79-84.

12. Njeri, S. G., Makumbi, D., Warburton, M. L., Jumbo, M. B., \& Chemining'wa, G. (2017). Genetic analysis of tropical quality protein maize (Zea mays L.) germplasm. Euphytica, 213(11), 119.

13. Onejeme, F. C., Okporie, E. O., \& Eze, C. E. (2020). Combining Ability and Heterosis in Diallel Analysis of Maize (Zea mays L.) Lines. International Annals of Science, 9(1), 188200.

14. Rohman, M. M., Hossain, M. G., Omy, S. H., Methela, N. J., \& Molla, M. R. (2019). Evaluation of diallel crosses of maize at multilocation (Zea mays L.) for saline tolerance. Journal of Cereals and Oilseeds, 10(2), 29-42.

15. Tilahun, B., Dida, M., Deresa, T., Garoma, B., Demissie, G., Kebede, D., ... \& Chere, A. T. (2017). Combining ability analysis of quality protein maize (QPM) inbred lines for grain yield, agronomic traits and reaction to grey leaf spot in mid-altitude areas of Ethiopia.

16. Ali, M. (2020). Diallel analysis of maize inbreds for grain yield, protein and tryptophan content. Egyptian Journal of Agronomy, 42(1), 117.

Cite This Article: Tefera Kumsa et al (2021). Evaluation of Diallel Crosses of Highland Adapted QPM Maize Hybrids Under Optimum and Low Nitrogen Conditions. EAS J Biotechnol Genet, 3(2), 30-37. 